\title{
Comparative transcriptome analyses of Pseudomonas aeruginosa
}

\author{
Deepak Balasubramanian' and Kalai Mathee ${ }^{2 *}$ \\ ${ }^{\prime}$ Department of Biological Sciences, College of Arts and Science, Florida International University, Miami, FL 33199, USA \\ ${ }^{2}$ Department of Molecular Microbiology and Infectious Diseases, College of Medicine, Florida International University, Miami, \\ FL 33199, USA \\ *Correspondence to: Tel: +1 305348 0628; Fax: +1 305348 2913; E-mail: Kalai.Mathee@fiu.edu
}

Date received: 5th May 2009

\begin{abstract}
One of the hallmarks of bacterial survival is their ability to adapt rapidly to changing environmental conditions. Niche adaptation is a response to the signals received that are relayed, often to regulators that modulate gene expression. In the post-genomic era, DNA microarrays are used to study the dynamics of gene expression on a global scale. Numerous studies have used Pseudomonas aeruginosa-a Gram-negative environmental and opportunistic human pathogenic bacterium - as the model organism in whole-genome transcriptome analysis. This paper reviews the transcriptome studies that have led to immense advances in our understanding of the biology of this intractable human pathogen. Comparative analysis of $23 P$. aeruginosa transcriptome studies has led to the identification of a unique set of genes that are signal specific and a core set that is differentially regulated. The 303 genes in the core set are involved in bacterial homeostasis, making them attractive therapeutic targets.
\end{abstract}

Keywords: alginate, bacteria, biofilm, comparative transcriptome, copper, iron limitation, microarray, osmotic stress, oxidative stress, pathogenesis, PCP, Pseudomonas aeruginosa, sulphate, quorum sensing

\section{Introduction}

Bacteria rapidly adapt to changing conditions by altering gene expression. The process of bacterial adaptation has fascinated researchers, as it provides important insights into their physiology. This information is especially useful for human pathogens because it can be applied to combating infections more effectively. This premise has led to studies that examine gene expression on a global scale. An entire set of RNA transcripts produced by a population of cells at any given time during gene expression is referred to as a transcriptome. ${ }^{1}$ Variations in the external environment or genome perturbations, such as mutations, can alter the transcriptome of a cell. Transcriptome studies thus provide us with a snapshot of the organism's response, based on the genes that are expressed.
Although there is not always a linear relationship between the expressed genes and the proteins synthesised, such studies give us a reasonable insight into the state of the cell.

In the pre-genomic era, transcriptome analysis relied on techniques such as differential display and RNA arbitrary primed polymerase chain reaction (PCR), ${ }^{2-4}$ cDNA-amplified restriction fragment length polymorphism, ${ }^{5-7}$ random expressed sequence tag sequencing, ${ }^{8}$ serial analysis of gene expression (SAGE), ${ }^{9}$ LongSAGE, ${ }^{10}$ SuperSAGE ${ }^{11,12}$ and massively parallel signature sequencing. ${ }^{13}$ Prior sequence information is not required for these methods but they are both labour- and time-intensive. Bacterial studies entered the genomic era with the sequencing of the Haemophilus influenzae genome in $1995 .{ }^{14}$ Since 
then, over 770 bacteria have been fully sequenced. Large-scale sequencing has ushered in the development of high-throughput genome-wide expression techniques such as DNA microarrays. This powerful technology has been harnessed in numerous studies, under diverse conditions, to elucidate whole-genome expression in both prokaryotes and eukaryotes. One such prokaryote is Pseudomonas aeruginosa, for which DNA microarrays have been of immense help in understanding its complex biology.

\section{P. aeruginosa}

P. aeruginosa is a Gram-negative human pathogen of the family Pseudomonadaceae and can switch from being an environmental isolate, found in the soil and water, to being a human pathogen. ${ }^{15}$ It is typical of opportunistic human pathogens, being implicated in a wide range of infections of the pulmonary tract, ${ }^{16,17}$ urinary tract, ${ }^{18-21}$ burns $^{22-24}$ and wounds, ${ }^{25,26}$ among others. It causes persistent nosocomial infections ${ }^{27,28}$ and is also a major pathogen in cystic fibrosis (CF) patients. One of the major weapons in its arsenal is its resistance to antibiotics. It is inherently resistant but can also acquire resistance genes from other bacteria, making it a notorious pathogen. Understandably, numerous studies over the years have focused on elucidating the mechanisms of pathogenesis and antibiotic resistance of $P$ aeruginosa. Since the sequencing of the genome, ${ }^{29}$ the emphasis of these studies has shifted from conventional pathogenesis research, where the focus was on individual virulence determinants, to a much larger scale, involving whole-genome analyses.

The prototypic reference strain of $P$ aeruginosa is PAO1, isolated from a burn wound, which was the first $P$ a aruginosa strain to be sequenced. ${ }^{29}$ The most prominent feature of the PAO1 genome is its size. It has one of the largest bacterial genomes, with 6.26 million base pairs, comprising almost 90 per cent protein-coding genes. The genome complexity of $P$. aeruginosa explains its ability to adapt and thrive in a wide range of environments. The genome has 5,570 open reading frames (ORFs), a number that is close to those of simple eukaryotes such as Saccharomyces cerevisiae. Other features of the PAO1 genome include the presence of four ribosomal RNA loci $(r r n)$, which are the regions of the longest repeats, and a high GC ratio, at 66.6 per cent. $^{29}$ Following the sequencing of the genome, high-density DNA microarrays were developed for $P$. aeruginosa ${ }^{30}$ that offered us a chance to take snapshots of the dynamic responses of this versatile genome to various stimuli. An important study in this context analysed the genomes of both environmental and clinical isolates of $P$. aeruginosa, ${ }^{31}$ and suggested the presence of a core genome in different strains and expression of different sets of genes to facilitate growth in particular environments. This calls for a robust control over transcription mediated by transcriptional regulators. Analysis revealed that about 10 per cent of the PAO1 genome comprises transcriptional regulators. ${ }^{29}$ This suggests the existence of a tight control over the way that genes are expressed in $P$ aeruginosa.

Subsequent to PAO1, other $P$. aeruginosa strains, such as PA14, ${ }^{32}$ PA2192 (a CF isolate), ${ }^{33}$ C3719 (the Manchester epidemic strain) ${ }^{34}$ and PACS2, ${ }^{35}$ have been sequenced. Comparative genome analysis of these five sequenced $P$. aeruginosa strains has led to the identification of 5,021 genes that are conserved between the strains (core gene set), the majority of which are housekeeping genes. ${ }^{35}$ In addition, there are at least 1,800 ORFs belonging to the accessory genome that comprises mostly hypothetical ORFs or those related to transposons and bacteriophages, and genes involved in niche adaptation specific to each of the five strains. ${ }^{35}$ The accessory set also contains transcriptional regulators that may also control expression of ORFs in the core genome. The currently available $P$ aeruginosa GeneChip (Affymetrix) is based on the PAO1 genome, which has 5,570 ORFs. In addition, this GeneChip contains probe sets for 117 ORFs from non-PAO1 strains, a gross under-representation of the accessory genome. Thus, performing microarray studies with non-PAO1 strains would seriously underestimate the number of genes in a transcriptional profile and would undoubtedly affect data interpretation. Thus the next major advance in 
P. aeruginosa microarrays would be the development of a pangenome array, comprising probe sets for the core and accessory genomes from all sequenced strains. This will greatly advance our understanding of the system by allowing researchers to study non-PAO1 strains and helping them better to appreciate the biology of this versatile bacterium.

\section{P. aeruginosa transcriptome studies}

\section{$P$. aeruginosa and iron limitation}

Iron is an important cofactor, which plays a critical role in many cellular processes. Low iron availability in host tissues is a major impediment that bacteria have to overcome in establishing an infection. Bacteria have evolved an elaborate system of acquiring iron from their surroundings. P. aeruginosa secretes siderophores (pyochelin and pyoverdine), which chelate iron and bring it back into the cell via specific cell-surface receptors. ${ }^{36}$ Fur is a major repressor that tightly regulates this uptake process by controlling the expression of the regulators, PvdS and PchR. ${ }^{37}$

In one of the initial transcriptome studies of the response of $P$ aeruginosa to iron, ${ }^{38}$ stationary phase $P$. aeruginosa cells were grown in the presence and absence of iron at $32^{\circ} \mathrm{C}$, followed by gene expression analysis. The results indicated that 137 genes were upregulated on iron depletion. ${ }^{38} \mathrm{~A}$ more systematic study was performed by Palma et al., who addressed how the transcriptome changes when iron-starved $P$. aeruginosa cells are fed with iron. ${ }^{39}$ Differential regulation was seen in the mRNA levels of 177 genes. Most of the genes were downregulated, especially genes involved in transport of small molecules, transcriptional regulators and hypothetical proteins. It was interesting to note that genes involved in virulence were upregulated, some of which probably help the cells to overcome the host innate immune system. ${ }^{39}$ An important finding was that 38 genes, including those coding for siderophore receptors, iron transport, regulators, sensors and sigma factors, were identified whose transcription decreased on iron treatment. These 38 genes were proposed to be part of a regulon involved in the iron response in
P. aeruginosa. In another study, Zheng and coworkers identified a hypothetical protein, PA2384, which regulates over 70 genes involved in the iron uptake process in a Fur-independent manner. ${ }^{40}$ Moreover, deleting PA2384 also led to differential expression of many genes involved in cellular physiology, such as translation, metabolism and electron transfer, indicating a broader role for this protein.

\section{P. aeruginosa and oxidative stress}

For bacteria successfully to infect a human host, they should be able to overcome many barriers. One primary defence mechanism is the production of reactive oxygen species (ROS) by the host cells that leads to oxidative stress in bacteria. Palma et al. studied the response of $P$. aeruginosa to a natural oxidant (hydrogen peroxide) in human systems, as an indication of the global transcriptional status of the cell's early response. ${ }^{41}$ The study confirmed that some of the genes that were earlier found to be important in a cell's response to oxidants were upregulated, in addition to identifying 520 genes with a significant (more than fivefold) change in mRNA levels. ${ }^{41}$ Of these, 216 genes were upregulated, seven of which (primarily hypothetical proteins of unknown function) were upregulated more than 50-fold. Other upregulated genes included cytotoxin genes such as exoenzymes $\mathrm{S}$ and $\mathrm{T}$ and PvdS, which control PrpL proteinase and exotoxin A expression and could potentially inhibit the cells of the innate immune system. Most of the genes that were downregulated are involved in metabolic pathways and ribosomal synthesis, which is logical, as under oxidative stress the cell would need first to deal with the stress, before actively metabolising or growing. ${ }^{41}$

In another study looking at the response to hydrogen peroxide, stationary phase cells of three strains belonging to two clonal types were tested. ${ }^{42}$ Two of the strains, TB and 892, were CF isolates and their response was compared with that of PAO1, which belongs to a different clonal type. The expression profiles of the CF isolates were more similar to each other than to PAO1, sharing more than 80 per cent of the differentially expressed genes with each other. This study 
highlighted the importance of clone-specific transcriptome profiles, and variations seen in both intraclonal (between the two CF isolates) and interclonal (between CF isolates and PAO1) strains.

Genome-wide transcriptomes of the three strains, $P$. aeruginosa $\mathrm{PAO} 1$ and the two $\mathrm{CF}$ isolates $\mathrm{TB}$ and 892, were also analysed for their response to superoxide-generating paraquat. ${ }^{43}$ Fewer than 30 genes were differentially expressed in each of the three strains, of which only six were upregulated in all three. Upregulated genes belonged to the following functional categories: oxidative stress response, transport, and acetyl coenzyme $\mathrm{A}$ and acetoin metabolism. Four genes of a single operon coding for the superoxide dismutase SodB, catalase KatA and alkyl hydroperoxide reductase AhpC were upregulated in three strains, highlighting their importance in the $P$. aeruginosa superoxide stress response. Energy metabolism genes were downregulated to counter the superoxide stress. ${ }^{43}$

Two recent papers by Small et al. ${ }^{44,45}$ looked at the oxidative stress response of $P$. aeruginosa. Both of these papers focused on the effect of oxidising agents routinely used in hospitals for surface sterilisation, and their effect on $P$. aeruginosa. The first was a transcriptome analysis of sodium hypochlorite $(\mathrm{NaOCl})$-induced oxidative stress. ${ }^{44}$ In this study, Luria-Bertani (LB) broth-grown mid-log phase P. aeruginosa $\mathrm{PAO} 1$ cells were treated for 20 minutes with sub-lethal concentrations of bleach. RNA extraction and microarray analysis followed. The results were compared with similarly grown untreated cells. About 20 per cent of the 5,570 genes of $P$. aeruginosa were differentially expressed in response to the treatment, approximately 10 per cent each being up- and downregulated. Responses similar to that seen with hydrogen peroxide were generated, with upregulation of catalase, alkyl hydroperoxide reductase and glutathione peroxide/ reductase genes indicating that the mode of action of bleach in inducing the oxidant defence system genes is similar to that of hydrogen peroxide. Genes involved in the transport of sulphur compounds were highly upregulated. A possible explanation for this observation is that the free electrons in the sulphydryl groups in the biological molecules react with the hypochlorous acid formed by exposure to $\mathrm{NaOCl}$, a known strong oxidiser, countering its effect. This would increase the chances of cell survival. ${ }^{44}$ Genes that are repressed are primarily those involved in oxidative phosphorylation and electron transport, indicating that the cells under $\mathrm{NaOCl}$-induced stress would have lowered energy owing to impaired metabolic processes.

Another study compared and analysed the transcription patterns of three different oxidative damage-inducing agents, $\mathrm{NaOCl}$, peracetic acid and hydrogen peroxide, on $P$. aeruginosa. ${ }^{45}$ Over 550, 300 and 200 genes were differentially regulated as a result of $\mathrm{NaOCl}$, peracetic acid and hydrogen peroxide, respectively. Of these, 63 genes were common to all three agents, 40 upregulated and 23 downregulated. About 25 per cent of the common upregulated genes were 'related to phage, transposon or plasmid' and eight per cent to membrane protein. The primary downregulated genes were those involved in the transport of small molecules. Other prominent gene categories included those encoding membrane proteins and putative enzymes. It is surprising to note that membrane protein genes were both up- and downregulated. $\mathrm{NaOCl}$ resulted in the greatest downregulation of genes involved in energy metabolism, suggesting that $\mathrm{NaOCl}$ may be more effective than the other two agents in limiting energy production. Peracetic acid exposure resulted in the stimulation of the expression of genes involved in adaptation and protection, and the downregulation of transcriptional regulators. Several protective mechanisms were triggered after exposure of cells to hydrogen peroxide. A significant finding of this study was the positive expression of the pyocin genes of the $\mathrm{F}-, \mathrm{R}-$ and S-types after hydrogen peroxide treatment but downregulation with the other two treatments. ${ }^{45}$ This has been linked to DNA damage and RecA activation, which has been known to induce pyocins. RecA has been demonstrated to cleave single-stranded DNA with the help of hydroxyl radicals that are formed by the exposure of cells to hydrogen peroxide. The finding that pyocin genes are induced in response to hydrogen peroxide treatment, published separately, ${ }^{46}$ demonstrates that 
$\mathrm{NaOCl}$ exposure leads to significant differences in the transcription pattern of $P$. aeruginosa and is more lethal to the cell than either hydrogen peroxide or peracetic acid. $^{45}$

Nde et al. tested the response of $P$. aeruginosa to ortho-phenylphenol (OPP), ${ }^{47}$ a widely used disinfectant and antimicrobial agent whose mode of action in bacteria has not yet been elucidated. Using two different exposure times (15 and 60 minutes), they identified genes coding for virulence, membrane and ribosomal proteins, upregulated at both time points. Forty-five genes involved in translation, post-translation modification and degradation were upregulated, primarily initiation, elongation and release factors. ${ }^{47}$ Genes encoding components of the $\mathrm{SecY}$ system involved in the export of various proteins, toxins and enzymes showed enhanced expression, as did some membrane-associated proteins involved in small molecule transport, possibly indicating that the OPP-treated bacteria were transporting proteins across the cell membrane as part of an adaptive process. Also upregulated were genes governing type IV pili assembly proteins, fatty acid biosynthesis and energy metabolism, all of which indicate adaptation. ${ }^{47}$

\section{P. aeruginosa and pentachlorophenol stress}

The response of $P$. aeruginosa to pentachlorophenol (PCP) was investigated recently. ${ }^{48}$ PCP is thought to embed in the membrane, where it acts as a proton shuttle uncoupling oxidative phosphorylation. ${ }^{49}$ In addition, since RpoS is the stationary phase sigma factor thought to be important in the cell's stress response, rpoS mutants were also examined under the same conditions. Sixty genes showed a twofold or greater increase in transcription. RpoS did not play a protective role in the cell's response to PCP-induced stress. The highlight of this study was the induction of multiple operons involved in multidrug efflux, the first anthropogenic induction of the MexAB-OprM system with chemicals. Notably, two operons of the resistance-nodulation-cell division (RND) pumps were upregulated, in addition to one operon that positively regulates MexABOprM. These are involved in antibiotic and solvent efflux. Putative major facilitator superfamily (MFS) proteins were also upregulated in response to PCP-induced stress. Selective downregulation of genes involved in central metabolism, fatty acid metabolism, energy metabolism and adaptation/ protection was observed. $^{48}$

\section{P. aeruginosa response to copper}

Copper is an essential micronutrient, and plays an important role in cellular respiration as part of the electron transport chain and many biological reactions. ${ }^{50}$ Bacteria have to control cellular levels of copper tightly, to prevent toxicity or starvation. Two studies looked at how $P$. aeruginosa responds to varying levels of copper.

Tietzel et al. identified 41 genes that form a core response of $P$. aeruginosa to copper, comparing transcriptomes of cells exposed to high levels of copper for a short time (copper-shocked, 405 genes) with those grown in copper (copper-adapted, 331 genes). ${ }^{51}$ Genes involved in the active efflux of copper included those encoding transporters, copper oxidase, periplasmic thiol-disulphide interchange proteins, lyases and transcriptional regulators.

Copper starvation of $P$. aeruginosa under aerobic conditions $^{52}$ led to downregulation of 132 genes, while only ten genes were upregulated. The most strongly downregulated genes were the iron-acquisition genes, mostly those repressed by Fur, the iron-uptake regulator. One of the downregulated transcriptional regulators, PA3410, encodes a probable extracellular sigma factor. ${ }^{51}$ The upregulated genes included mostly hypothetical genes and a bacterioferritin involved in iron storage. In fact, copper starvation and iron depletion ${ }^{38,39}$ led to the expression of similar sets of genes but have opposite effects on them.

\section{P. aeruginosa and sulphate starvation}

The CF lung mucin is highly sulphated, probably as a defence mechanism to prevent degradation by bacterial enzymes. ${ }^{53,54}$ The metabolically versatile $P$ aeruginosa can utilise a variety of organic and inorganic compounds for growth. Under limiting 
conditions of inorganic sulphate, the cells can take up organosulphur compounds for growth without affecting the growth rate. ${ }^{55}$ The global response to sulphate starvation in a non-mucoid CF isolate of P. aeruginosa, E601, and PAO1 was addressed. ${ }^{56}$ The study was aimed at determining which genes are regulated by sulphur supply and whether this list was altered during CF infection, a condition simulated by exposure to mucin. Three groups of responses were observed: response to sulphur starvation, to mucin as a sulphur source and to mucin exposure. Functional analysis of differentially expressed genes revealed that both strains showed similar responses in the different functional classes, with maximal differential regulation of genes encoding to hypothetical proteins, transport of small molecules and putative enzymes. Unlike PAO1, the CF isolate E601 was shown to utilise mucin as a sulphur source. Moreover, several genes involved in the type III secretion system and exoenzyme $\mathrm{S}$ were induced, suggesting that cells recognise mucin not only as a sulphur source, but also as a host component: a dual response.

\section{P. aeruginosa and osmotic stress}

$P$. aeruginosa, being a versatile pathogen, can survive in a wide range of environments, some of which can be osmotically stressful. Transcriptional profiling of osmotically stressed $P$ aeruginosa was studied. ${ }^{57}$ P. aeruginosa PA14 cells, grown in glucose minimal media, were exposed to osmotic stress, either under steady-state conditions $(0.3 \mathrm{M} \mathrm{NaCl}$ and $0.7 \mathrm{M}$ sucrose) or osmotically up-shocked (0.3 $\mathrm{M} \mathrm{NaCl}$ for a short time). A major difference in the expression pattern between these two conditions is the number of genes that are differentially regulated. While 431 genes were differentially regulated on osmotic up-shocking, only about 100 genes were more than threefold regulated under either condition of steady-state osmotic shock. These studies led to the identification of a steady-state osmotic shock regulon comprising 66 genes. Forty of these are virulence associated, primarily genes of the type III secretion system, two are type III exotoxins and two are chaperones. By contrast, the osmotically up-shocked cells showed repression of the type III secretion system genes that the authors hypothesise to be an energysaving step. In addition, the sigma factor $A \lg T / \mathrm{U}$, previously known to be induced in various stress conditions, and genes known to be involved in osmoprotection, including hydrophilins, whose exact role in osmoprotection has not yet been conclusively proven in bacteria, ${ }^{58}$ were induced in all the conditions of osmotic shocking. ${ }^{57}$

\section{$P$ aeruginosa and quorum sensing}

$P$. aeruginosa senses the cell density for coordinated expression of virulence factors using a global regulatory system called quorum sensing (QS). Sensing is achieved using small diffusible molecules that in $P$. aeruginosa and other Gram-negative bacteria are the N-acylated homoserine lactones. ${ }^{59}$ P. aeruginosa has two N-acylated homoserine lactone systems, las and $r h l .^{60}$ Transcriptome analysis of las I rhlI mutants were performed by three groups. ${ }^{61-63}$ Two of these groups $^{61,62}$ identified about 300 genes that were induced by QS, while the other group found only 163 differentially expressed genes as a result of use of more stringent criteria. The three studies had approximately 100 common genes and the encoded products belonged to the functional class of secreted factors. This group included toxins and extracellular enzymes. In addition, above-average expression changes were seen in the genes involved in metabolism, biosynthesis of cofactors and fatty acid metabolism, implying that QS signals mediated by $\mathrm{N}$-acylated homoserine lactones were involved in the regulation of many genes other than those involved in virulence. The third group, Hentzer et al., targeted the QS system in an attempt to mitigate $P$. aeruginosa virulence ${ }^{63}$ using a furanone isolated from the Australian seaweed Delisea pulchra. The authors elegantly showed, using transcriptome analyses, that about 80 per cent of the genes that were downregulated by the furanone were also repressed in an $\mathrm{N}$-acylated homoserine lactones mutant. The furanone compound also affected biofilm formation and the persistence of infection in a mouse lung model. Together, the three studies led to the identification of a core set of 77 genes of 
the QS regulon that are positively regulated. ${ }^{63}$ Schuster and Greenberg reviewed many other studies of regulators that further elucidate the complex QS system and the intricate interconnections. $^{64}$ They propose a complex network that involves 13 different regulators that interact with LasI and RhlI and form the QS regulon.

\section{P. aeruginosa biofilms}

Quorum sensing has been implicated as an important step in the formation of ordered, antibiotic-resistant bacterial communities called biofilms. ${ }^{65,66}$ Planktonic cells, in response to external stimuli, form biofilms in varied habitats, such as in the CF lung, on surgical implants and in various environmental situations, exhibiting drastically altered physiology; this led to the hypothesis that the gene expression between these two states of cells are very different. One of the earliest studies that employed microarrays to understand differences in gene expression patterns between planktonic and biofilm cells, in addition to antibiotic resistance mechanisms in biofilms, was by Whiteley et al. ${ }^{67}$ Using chemostats, it was found that a surprisingly small number (1 per cent) of genes were differentially regulated between planktonic and biofilm cells. The most highly upregulated genes were those of the Pf1-like prophage, with nine of the 11 genes on the chromosome induced over 1001,000 fold in the biofilm cells. This could imply higher rates of gene transfer in biofilms via transduction. Genes involved in bacterial motility were repressed, as was rpoS, the stationary phase sigma factor. Strains harbouring a mutant rpoS were shown to form larger, more drug-resistant biofilms. Exposure of the biofilm to the antibiotic tobramycin, followed by transcriptional analysis, showed that $\operatorname{tol} A$ was upregulated, which can serve as a barrier to aminoglycoside uptake. Cytochrome C oxidase genes were repressed, probably slowing growth and negating the antibiotic effect. In addition, antibiotic exposure also induced two probable efflux systems and many hypothetical genes.

The effect of the $\beta$-lactam imipenem on gene expression in planktonic and biofilm cells of
P. aeruginosa was examined by Bagge et al. ${ }^{68}$ Microarray analysis of imipenem-exposed planktonic and biofilm cells revealed that the chromosomal $\beta$-lactamase ampC was overexpressed in these cells under these conditions. Thirty-four genes were more than fivefold differentially regulated and included genes involved in alginate production, flagella biosynthesis, cell division, and peptidoglycanrelated and type 4 pili biogenesis. In addition, biofilm cells also showed enhanced production of alginate, unlike in the Whiteley study with tobramycin. Bagge et al. could not ascribe higher antibiotic resistance of biofilm cells to a specific set of genes owing to similar expression patterns in planktonic and biofilm cells. ${ }^{68}$

A more recent study made a comprehensive analysis of transcriptomes of planktonic cultures (in $\log$ and stationary phase) and biofilms at eight, 14, 24 and 48 hours (microcolony and mature biofilms) using a static biofilm model. ${ }^{67}$ This study showed a similar gene expression pattern between developing biofilms and log phase planktonic cultures, while mature biofilms had expression patterns similar to stationary phase cells. The most highly upregulated genes in biofilm cells were those of a Pf1-related temperate bacteriophage, suggesting an important role for phage-mediated gene transfer in biofilm cells. Downregulated genes include those coding for pili, which suggests that, although pili are required for the establishment of biofilms, they are not essential in mature biofilms. Also, expression is relatively stable in developed biofilms, with only 1 per cent of the genes differentially expressed. This was the first study to look at global gene expression for two planktonic and two biofilm states of the cells, and the authors have generated a comprehensive dataset for reference that compares transcriptomes of planktonic, developing and mature biofilms.

\section{Transcriptomics of alginate production in P. aeruginosa}

Alginate gene upregulation in $P$ aeruginosa is one of the primary modes of survival in the CF lung and converts the non-mucoid cells to a mucoid phenotype $^{69}$ as a result of over-production of the 
extracellular polysaccharide alginate. Production of alginate is part of a stress response in $P$. aeruginosa and is mediated by the alternate sigma factor $\mathrm{AlgT} / \mathrm{U}$, a sigma $\mathrm{E}$ homologue. AlgT/U is held sequestered by the anti-sigma factor MucA. Release of AlgT/U leads to alginate production and is part of a much larger response to help the cells adapt. ${ }^{70}$ Transcriptome analysis of muсA, algT/U-mucA mutants and the wild-type PAO1 showed that alginate was upregulated in the mucA mutant when there was no control on AlgT/U production. This control was lost in the double mutant, as expected. $^{71}$ Interestingly, many genes responsible for toxin production, enzymes and transport were also upregulated, indicating that $\mathrm{AlgT} / \mathrm{U}$ was part of a regulon that coordinated the expression of many genes, ultimately enhancing $P$. aeruginosa survival in the harsh CF lung environment.

$P$ aeruginosa, like other bacteria, produces flagella only when needed, for example in biofilm formation, and has been implicated in establishing pulmonary infections. ${ }^{72}$ AlgT/U was shown negatively to regulate flagella production in $P$. aeruginosa by downregulating an essential flagella regulator gene, FleQ. ${ }^{73}$ There are many regulators in flagella biosynthesis, and a systematic study of several regulator mutants was performed by Dasgupta et al. ${ }^{74}$ Using transcriptome analysis, they found that flagella are assembled in a four-step process that is controlled by the sequential expression of different regulators.

\section{Lipopolysaccharide as a virulence determinant}

Lipopolysaccharides play a major role in $P$. aeruginosa virulence. ${ }^{75}$ The bacteria are known to modify their lipopolysaccharide layer in response to the milieu. Lipid A, a major constituent of the bacterial liposaccharide, is hepta- or hexa-acetylated in strains causing acute infections, while it is penta-acetylated in chronic infections such as $\mathrm{CF}^{76}$ Lipopolysaccharide changes with alginate production, ${ }^{77}$ and production of both of these important virulence factors are influenced by oxygen concentration. ${ }^{78-80}$ Although we are not aware of any microarray studies that compare lipopolysaccharide mutants, Firoved et al. compared the expression patterns of a mucoid (PAO578II) and the isogenic non-mucoid parent (PAO6865), and demonstrated that lipoprotein genes are most highly upregulated when $P$ aeruginosa switches over to the mucoid phenotype. ${ }^{81}$ Eight of the nine genes that were upregulated over 30-fold encoded lipoproteins, which can have toxic effects in environments such as the CF lung by disrupting host Toll-like receptor 2 signalling and are also antigenic, activating host defence mechanisms. Thus, some of these lipoproteins have been termed lipotoxins. ${ }^{81}$ Elucidating mechanisms of co-regulation of lipopolysaccharide production/modification and conversion of the strains to mucoidy would help us better to understand the adaptation/pathogenesis of this versatile bacterium in chronic infections such as CF.

\section{Transcriptome of a post-transcriptional regulator}

Burrowes et al. studied the role of a small posttranscriptional regulatory protein, RsmA. ${ }^{82} \mathrm{RsmB}$, a small untranslated regulatory RNA, binds to RsmA, limiting its activity. ${ }^{83}$ RsmA controls the expression of many virulence genes and QS signalling molecule synthesis, ${ }^{83,84}$ and thus plays an important role in $P$ aeruginosa pathogenesis. Although some of the targets of RsmA were known, this study focused on determining the direct and indirect regulatory control exerted by RsmA in P. aeruginosa. ${ }^{82}$ Transcriptional analysis of PAO1 and the $\operatorname{rsm} A$ mutant revealed that, of the 506 genes that had significantly altered expression, genes involved in iron acquisition and antibiotic resistance, especially those involved in the MexEFOprN pump, were upregulated and genes involved in Pseudomonas quinolone signal biosynthesis and motility were downregulated. The data obtained suggest that $\mathrm{RsmA}$ is a global regulator. ${ }^{82}$

\section{P. aeruginosa interactions with human cells}

When a bacterium comes into contact with a host cell for the first time, there is a drastic change in the lifestyle of the bacteria which aids in 
colonisation. The host, on the other hand, recognises this threat and mounts an immune response. As one might predict, this race between the pathogen and host to outdo each other will undoubtedly be accompanied by changes in the gene expression profile in both organisms. Numerous studies have looked at the response of the human immune system to $P$ aeruginosa exposure, and we have a fairly good idea of the immune signals and molecules involved. One of the first studies to employ transcriptomics to investigate host responses to initial contact with P. aeruginosa was carried out by Ichikawa et al. ${ }^{85}$ Using a time course microarray experiment with lung epithelial cells exposed to $P$ aeruginosa and custom-made cDNA microarrays containing about 1,500 human clones with both characterised and uncharacterised genes, they determined that about 25 per cent of the human genes represented on the array were differentially regulated. Of interest was a lipopolysaccharide-independent twofold activation of the interferon regulatory factor- 1 gene, with no activation of other downstream genes that are normally activated by interferon. This indicated that $P$ aeruginosa activation of interferon regulatory factor-1 is different from the normal mechanism of interferon regulatory factor- 1 activation mediated by cognate receptor binding. ${ }^{85}$ Using a type IV pili mutant, these authors demonstrated that PilA is the ligand responsible for lipopolysaccharide-independent activation of interferon regulatory factor- 1 . This study demonstrated the power of microarrays in understanding host-pathogen interactions by elucidating potentially new signalling pathways and characterising the roles of different virulence factors involved in pathogen recognition and mounting an immune response.

Two other studies have employed DNA microarrays to study the interactions of $P$. aeruginosa to human epithelial cells, but the results are contradictory with respect to the regulation of iron-responsive genes. In the first study, by Frisk et al., ${ }^{86}$ P. aeruginosa was exposed to primary human airway epithelial cells for four and 12 hours in vitro and transcription was analysed post-exposure. Two major groups of genes were differentially regulated: phosphate uptake genes were upregulated, whereas iron-acquisition genes were downregulated. This was contrary to the notion that iron is an important limiting factor in the human host; one would have expected upregulation of the iron-acquisition genes. The authors concluded that $P$. aeruginosa was able to acquire enough iron for infection that led to deregulation of those genes. The reverse was true for phosphate.

In an attempt better to understand the initial adaptation processes of $P$ aeruginosa, especially on interaction with human epithelia, a study by Chugani and Greenberg ${ }^{87}$ compared transcriptomes of $P$ aeruginosa $\mathrm{PAO} 1$ wild-type with a double mutant that was deficient in the type III secretion system and rhamnolipid biosynthesis. These two bacterial systems are key factors contributing to human airway epithelial cell damage. Strain-specific responses were seen. Whereas virulence genes such as PA-1 galactophilic lectin were induced in the wild-type strain, alginate regulatory genes were induced in the mutant, along with $\operatorname{gac} A,{ }^{87}$ which is known to be upregulated in chronic $P$. aeruginosa infections. ${ }^{30}$ Also, Vfr-a known upregulated regulator in acute infections - was downregulated, indicating the early stages of a chronic infection.

In contrast to the results obtained by Frisk et al., ${ }^{86}$ the Chugani and Greenberg study showed that ironstarvation genes were positively regulated, indicating that the infecting cells had little iron to utilise. This is in concordance with the belief that infecting bacterial cells have little access to iron in the human body. One possible explanation for these discordant results, as noted by the authors, is that the epithelial cell integrity in the previous study could have been compromised, leading to leakage of intracellular iron and resulting in iron-replete conditions. This would explain the increased expression of iron-dependent genes over time seen in the Frisk study. Epithelial cell integrity was monitored in the Chugani and Greenberg study by measuring the transepithelial electrical resistance $(\mathrm{Rt})$. An interesting finding by Chugani et al. is that the general pattern of gene expression is similar to that seen in CF isolates from chronic human infections.

Many bacteria use type III secretion systems to inject proteins directly into host cells. An interesting whole-genome array study by Wolfgang et al. ${ }^{31}$ 
looked at the conditions and regulatory mechanisms that control the expression of the type III secretion systems. They utilised the observation that type III secretion systems are induced by either growth in low-calcium-containing medium or by cell contact, and, by growing cells in low calcium, found 11 calcium-dependent regulators that were involved in the process. Analysis of these proteins led to the discovery that a membrane-associated adenylate cyclase initiates a signalling pathway that controls the expression of type III secretion system genes. ${ }^{31}$

The transcriptome studies of $P$ aeruginosa with human cells indicate the presence of as yet unidentified signals, from both the host and the bacteria, during interactions. Unravelling these signals could provide us with valuable information effectively to counter infections by $P$. aeruginosa: microarrays will undoubtedly prove vital in this endeavour.

\section{Comparative analysis of all transcriptomes}

In order better to understand the response of $P$ aeruginosa to the different stress conditions, a comparison was carried out of significantly differentially regulated genes from the studies within each stress condition. This analysis was performed only for conditions for which there had been multiple studies and publicly available gene lists. This comparative analysis led to the identification of common sets of genes that are regulated in response to each particular stress condition across the different studies. Additionally, unique genes that are specifically regulated only in individual studies were also identified. These data are summarised in Table 1.

Analysis of significantly differentially regulated genes from all of the above studies led to the identification of 303 genes that form a core set and are differentially expressed in all the different conditions (Table S1). This suggests that this set of core genes is important for maintaining homeostasis in $P$. aeruginosa. Functional categorisation of the core set genes (Figure S1) reveals that, apart from the hypothetical genes ( 27 per cent), the most highly differentially regulated genes encode membrane proteins (15 per cent), followed by genes
Table I. Comparative analysis of significantly differentially regulated genes from various studies within each condition

\begin{tabular}{|lccc|}
\hline & & \multicolumn{2}{c}{$\begin{array}{c}\text { Differentially } \\
\text { regulated genes }\end{array}$} \\
\hline $\begin{array}{l}\text { Stress } \\
\text { condition }\end{array}$ & $\begin{array}{c}\text { Number of } \\
\text { studies }\end{array}$ & Common & Unique \\
\hline Iron & 3 & 69 & 220 \\
\hline $\begin{array}{l}\text { Quorum } \\
\text { sensing }\end{array}$ & 3 & 121 & 862 \\
\hline Copper & 2 & 26 & 209 \\
\hline $\begin{array}{l}\text { Oxidative } \\
\text { stress }\end{array}$ & 7 & 83 & 386 \\
\hline Biofilms & 3 & 55 & 650 \\
\hline
\end{tabular}

involved in the transport of small molecules (11 per cent), adaptation and protection ( 7 per cent) and transcriptional regulators (6 per cent), clearly indicating an adaptive response of $P$. aeruginosa to the changing environment. This microarray core set of 303 genes was then compared with the core genome of $P$ aeruginosa, which contains genes found in all $P$. aeruginosa sequenced strains. ${ }^{35}$ Most of these genes (90 per cent) belong to the P. aeruginosa core genome, but 29 genes belonging to the PAO1 accessory genome were also identified (Table S1). This set of 29 genes contains two type IV pili-related genes, genes involved in adaptation and protection (membrane proteins, transport of small molecules and metabolism) and hypothetical genes, among others. A caveat to this analysis is that, unlike PAO1, the other sequenced P. aeruginosa genomes do not have 100 per cent coverage.

After subtracting the core set, sets of unique genes that are specifically differentially regulated in each of the conditions examined were compiled. Functional categorisation of all the genes in the unique gene sets (Table S2) revealed that, overall, as expected, hypothetical genes were the most differentially regulated. In addition, genes involved in adaptation (membrane proteins, transport of small molecules, putative enzymes, transcriptional regulators and energy metabolism) showed the highest percentage of differential regulation. 
Table S3 lists all the unique genes identified under the various conditions used in these studies. In the iron studies, for example, genes of the secreted factors group (encoding toxins, enzymes, alginate) constitute 7 per cent of the differentially regulated genes. This supports the view that $P$ aeruginosa upregulates virulence genes in response to iron limitation, to help survival in the host system. $^{39}$ Analysis of the data from response of $P$. aeruginosa to copper revealed that 33 per cent of the differentially regulated genes encode membrane proteins, a much higher percentage than for small molecule transport genes (19 per cent) or hypothetical genes (14 per cent), suggesting that copper stress primarily affects the membrane. By far the most interesting observation was from the osmotic shock study. In stark contrast to the other studies, in this study there was only a small percentage of hypothetical genes ( 3 per cent) that were differentially regulated. ${ }^{57}$ Transcriptional regulators (37 per cent), protein secretion/export apparatus (33 per cent) and two-component regulatory systems (10 per cent) form over 70 per cent of the genes differentially regulated under osmotic stress. The osmotic study was performed using $P$. aeruginosa PA14, which is known to be more virulent than $P$ aeruginosa PAO1 in a wide range of hosts. ${ }^{8-90}$ Since the gene chips used in the Aspedon et al's osmotic study were those designed for PAO1 genome, which has 5,570 ORFs, compared with 5,905 in PA14-there could be an under-representation of the genes (potentially 335 genes that are present in PA14 and not in PA01) that are differentially regulated.

\section{Conclusions}

The transcriptome analysis studies that have been reviewed here provide evidence of the immense power of the DNA microarray. Microarray profiling generates an enormous amount of data that add to our knowledge database. Genes that are differentially regulated by transcription factors, by the host environment, by changing physiological conditions (including the response to external stimuli, which could range from nutrients to other factors affecting growth) and by interactions with host cells have all been studied by microarrays. A previous review by Goodman and Lory ${ }^{30}$ involving nine P. aeruginosa microarray studies identified about a quarter of the genome to be differentially regulated in response to various stimuli. The present authors' comparative analysis of the significantly differentially regulated genes from all of the various studies showed that about 35 per cent of the $P$ aeruginosa genome $(1,932$ genes $)$ is regulated overall. To arrive at a more comprehensive understanding of how bacteria react to the ever-changing environment, physiological experiments need to be performed, with appropriate analysis of data, protocol standardisation and appropriate controls put in place.

The next major advances in DNA microarrays would be to develop technologies to analyse the changing transcriptome of the host-pathogen interaction process in vivo, and pangenome arrays that would give us insights into the workings of this versatile bacterium. Together, these will help us to devise ways better to counter $P$ aeruginosa infections.

\section{Acknowledgments}

This work is partly supported by NIH SC1 GM083677 (K.M.) and Florida International University Teaching Assistantship (D.B.). We acknowledge Dr Lisa Schneper and Dr Giri Narasimhan for critical reading of the manuscript and Camilo Valdes for assistance with the PAO1 core genome analysis.

\section{References}

1. Kellam, P. (2000), 'Host-pathogen studies in the post-genomic era', Genome Biol. Vol. 1, reviews 1009.1-1009.4.

2. Liang, P. and Pardee, A.B. (1995), 'Recent advances in differential display', Curr. Opin. Immunol. Vol. 7, pp. 274-280.

3. Liang, P. and Pardee, A.B. (1992), 'Differential display of eukaryotic messenger RNA by means of the polymerase chain reaction', Science Vol. 257, pp. 967-971.

4. Welsh, J., Chada, K., Dalal, S.S., Cheng, R. et al. (1992), 'Arbitrarily primed PCR fingerprinting of RNA', Nucleic Acids Res. Vol. 20, pp. 4965-4970.

5. Vos, P., Hogers, R., Bleeker, M., Reijans, M. et al. (1995), 'AFLP: A new technique for DNA fingerprinting', Nucleic Acids Res. Vol. 23, pp. 4407-4414.

6. Bachem, C.W., van der Hoeven, R.S., de Bruijn, S.M., Vreugdenhil, D. et al. (1996), 'Visualization of differential gene expression using a novel method of RNA fingerprinting based on AFLP: Analysis of gene expression during potato tuber development', Plant J. Vol. 9, pp. $745-753$.

7. Brugmans, B., Fernandez del Carmen, A., Bachem, C. W., van Os, H. et al. (2002), 'A novel method for the construction of genome wide transcriptome maps', Plant J. Vol. 31, pp. 211-222. 
8. Kamoun, S., Hraber, P., Sobral, B., Nuss, D. et al. (1999), 'Initial assessment of gene diversity for the oomycete pathogen Phytophthora infestans based on expressed sequences', Fungal Genet. Biol. Vol. 28, pp. 94-106.

9. Velculescu, V.E., Zhang, L., Zhou, W., Vogelstein, J. et al. (1997), 'Characterization of the yeast transcriptome', Cell Vol. 88, pp. 243-251.

10. Saha, S., Sparks, A.B., Rago, C., Akmaev, V. et al. (2002), 'Using the transcriptome to annotate the genome', Nat. Biotechnol. Vol. 20, pp. 508-512.

11. Matsumura, H., Ito, A., Saitoh, H., Winter, P. et al. (2005), 'SuperSAGE', Cell Microbiol. Vol. 7, pp. 11-18.

12. Matsumura, H., Reich, S., Ito, A., Saitoh, H. et al. (2003), 'Gene expression analysis of plant host-pathogen interactions by SuperSAGE', Proc. Natl. Acad. Sci. USA Vol. 100, pp. 15718-15723.

13. Brenner, S., Johnson, M., Bridgham, J., Golda, Q. et al. (2000), 'Gene expression analysis by massively parallel signature sequencing (MPSS) on microbead arrays', Nat. Biotechnol. Vol. 18, pp. 630-634.

14. Fleischmann, R.D., Adams, M.D., White, O., Clayton, R.A. et al. (1995), 'Whole-genome random sequencing and assembly of Haemophilus influenzae Rd', Science Vol. 269, pp. 496-512.

15. Holloway, B.W. and Morgan, A.F. (1986), "Genome organization in Pseudomonas', Annu. Rev. Microbiol. Vol. 40, pp. 79-105.

16. Grassme, H., Jin, J., Wilker, B., von Kurthy, G. et al. (2006), 'Regulation of pulmonary Pseudomonas aeruginosa infection by the transcriptional repressor Gfi1', Cell Microbiol. Vol. 8, pp. 1096-1105.

17. Gregory, A.D., Hogue, L.A., Ferkol, T.W. and Link, D.C. (2007), 'Regulation of systemic and local neutrophil responses by G-CSF during pulmonary Pseudomonas aeruginosa infection', Blood Vol. 109, pp. 3235-3243.

18. Rodriguez, C.N., Molina, N., Garcia, A., Nino, C.R. et al. (2002), 'Comparative study of antimicrobial resistance of Pseudomonas aeruginosa strains isolated from urinary tract infection in patients from Caracas and Lima', Int. J. Antimicrob. Agents Vol. 20, pp. 476-477.

19. Shigemura, K., Arakawa, S., Sakai, Y., Kinoshita, S. et al. (2006), 'Complicated urinary tract infection caused by Pseudomonas aeruginosa in a single institution (1999-2003)', Int. J. Urol. Vol. 13, pp. 538-542.

20. Sobczyk, D., Krynicki, T., Blumczynski, A., Zaniew, M. et al. (2006), 'New, successful treatment of urinary tract infection caused by Pseudomonas aeruginosa', Prz. Lek. Vol. 63 (Suppl. 3), pp. 140-141.

21. Takeyama, K., Kunishima, Y., Matsukawa, M., Takahashi, S. et al. (2002), 'Multidrug-resistant Pseudomonas aeruginosa isolated from the urine of patients with urinary tract infection', J. Infect. Chemother. Vol. 8, pp. 59-63.

22. Dale, R.M., Schnell, G. and Wong, J.P. (2004), 'Therapeutic efficacy of 'nubiotics' against burn wound infection by Pseudomonas aeruginosa', Antimicrob. Agents Chemother. Vol. 48, pp. 2918-2923.

23. Estahbanati, H.K., Kashani, P.P. and Ghanaatpisheh, F. (2002), 'Frequency of Pseudomonas aeruginosa serotypes in burn wound infections and their resistance to antibiotics', Burns Vol. 28, pp. 340-348.

24. McVay, C.S., Velasquez, M. and Fralick, J.A. (2007), 'Phage therapy of Pseudomonas aeruginosa infection in a mouse burn wound model', Antimicrob. Agents Chemother. Vol. 51, pp. 1934-1938.

25. Johnstone, I.E. (1977), 'Pseudomonas aeruginosa and wound infections', Nurs. Times Vol. 73(34) (Suppl. 55).

26. Secher, I., Hermes, I., Pre, S., Carreau, F. et al. (2005), 'Surgical wound infections due to Pseudomonas aeruginosa in orthopedic surgery', Med. Mal. Infect. Vol. 35, pp. 149-154.

27. Armour, A.D., Shankowsky, H.A., Swanson, T., Lee, J. et al. (2007), 'The impact of nosocomially-acquired resistant Pseudomonas aeruginosa infection in a burn unit', J. Trauma Vol. 63, pp. 164-171.

28. Marra, A.R., Bar, K., Bearman, G.M., Wenzel, R.P. et al. (2006), 'Systemic inflammatory response syndrome in adult patients with nosocomial bloodstream infection due to Pseudomonas aeruginosa', J. Infect. Vol. 53 , pp. $30-35$.

29. Stover, C.K., Pham, X.Q., Erwin, A.L., Mitoguchi, S.D. et al. (2000), 'Complete genome sequence of Pseudomonas aeruginosa PA01, an opportunistic pathogen', Nature Vol. 406, pp. 959-964.

30. Goodman, A.L. and Lory, S. (2004), 'Analysis of regulatory networks in Pseudomonas aeruginosa by genomewide transcriptional profiling', Curr. Opin. Microbiol. Vol. 7, pp. 39-44.
31. Wolfgang, M.C., Lee, V.T., Gilmore, M.E. and Lory, S. (2003), 'Coordinate regulation of bacterial virulence genes by a novel adenylate cyclase-dependent signaling pathway', Dev. Cell Vol. 4, pp. 253-263.

32. Lee, D.G., Urbach, J.M., Wu, G., Liberati, N.T. et al. (2006), 'Genomic analysis reveals that Pseudomonas aeruginosa virulence is combinatorial', Genome Biol. Vol. 7, p. R90.

33. Pier, G.B., Matthews, W.J. Jr. and Eardley, D.D. (1983), 'Immunochemical characterization of the mucoid exopolysaccharide of Pseudomonas aeruginosa', J. Infect. Dis. Vol. 147, pp. 494-503.

34. Jones, A.M., Govan, J.R., Doherty, C.J., Dodd, M.E. et al. (2001), 'Spread of a multiresistant strain of Pseudomonas aeruginosa in an adult cystic fibrosis clinic', Lancet Vol. 358, pp. 557-558.

35. Mathee, K., Narasimhan, G., Valdes, C., Qiu, X. et al. (2008), 'Dynamics of Pseudomonas aeruginosa genome evolution', Proc. Natl. Acad. Sci. USA Vol. 105, pp. 3100-3105.

36. Cornelis, P. and Matthijs, S. (2007), Pseudomonas Siderophores and their Biological Significance, Berlin, Springer.

37. Ochsner, U.A., Vasil, A.I. and Vasil, M.L. (1995), 'Role of the ferric uptake regulator of Pseudomonas aeruginosa in the regulation of siderophores and exotoxin A expression: Purification and activity on ironregulated promoters', J. Bacteriol. Vol. 177, pp. 7194-7201.

38. Ochsner, U.A., Wilderman, P.J., Vasil, A.I. and Vasil, M.L. (2002), 'GeneChip expression analysis of the iron starvation response in Pseudomonas aeruginosa: Identification of novel pyoverdine biosynthesis genes', Mol. Microbiol. Vol. 45, pp. 1277-1287.

39. Palma, M., Worgall, S. and Quadri, L.E. (2003), 'Transcriptome analysis of the Pseudomonas aeruginosa response to iron', Arch. Microbiol. Vol. 180, pp. 374-379.

40. Zheng, P., Sun, J., Geffers, R. and Zeng, A.P. (2007), 'Functional characterization of the gene PA2384 in large-scale gene regulation in response to iron starvation in Pseudomonas aeruginosa', J. Biotechnol. Vol. 132, pp. $342-352$.

41. Palma, M., DeLuca, D., Worgall, S. and Quadri, L.E. (2004), 'Transcriptome analysis of the response of Pseudomonas aeruginosa to hydrogen peroxide', J. Bacteriol. Vol. 186, pp. 248-252.

42. Salunkhe, P., Topfer, T., Buer, J. and Tummler, B. (2005), 'Genomewide transcriptional profiling of the steady-state response of Pseudomonas aeruginosa to hydrogen peroxide', J. Bacteriol. Vol. 187, pp. 2565-2572.

43. Salunkhe, P., von Götz, F., Weihlmann, L., Lauber, J. et al. (2002), 'GeneChip expression analysis of the response of Pseudomonas aeruginosa to paraquat-induced superoxide stress', Genome Lett. Vol. 1, pp. $165-174$.

44. Small, D.A., Chang, W., Toghrol, F. and Bentley, W.E. (2007), 'Toxicogenomic analysis of sodium hypochlorite antimicrobial mechanisms in Pseudomonas aeruginosa', Appl. Microbiol. Biotechnol. Vol. 74, pp. 176-185.

45. Small, D.A., Chang, W., Toghrol, F. and Bentley, W.E. (2007), 'Comparative global transcription analysis of sodium hypochlorite, peracetic acid, and hydrogen peroxide on Pseudomonas aeruginosa', Appl. Microbiol. Biotechnol. Vol. 76, pp. 1093-1105.

46. Chang, W., Small, D.A., Toghrol, F. and Bentley, W.E. (2005), 'Microarray analysis of Pseudomonas aeruginosa reveals induction of pyocin genes in response to hydrogen peroxide', BMC Genomics Vol. 6, p. 115.

47. Nde, C.W., Jang, H.J., Toghrol, F and Bentley, W.E. (2008), 'Toxicogenomic response of Pseudomonas aeruginosa to orthophenylphenol', BMC Genomics Vol. 9, p. 473.

48. Muller, J.F, Stevens, A.M., Craig, J. and Love, N.G. (2007), 'Transcriptome analysis reveals that multidrug efflux genes are upregulated to protect Pseudomonas aeruginosa from pentachlorophenol stress', Appl. Environ. Microbiol. Vol. 73, pp. 4550-4558.

49. Escher, B.I., Hunziker, R. and Schwarzenbach, R.P. (1999), 'Kinetic model to describe the intrinsic coupling activity of substituted phenols in energy transducing membranes', Environ. Sci. Technol. Vol. 33, pp. $560-570$.

50. Frieden, E., Osaki, S. and Kobayashi, H. (1965), 'Copper proteins and oxygen. Correlations between structure and function of the copper oxidases', J. Gen. Physiol. Vol. 49(1) (Suppl. 213-252). 
51. Teitzel, G.M., Geddie, A., De Long, S.K., Kirisits, M.J. et al. (2006), 'Survival and growth in the presence of elevated copper: Transcriptional profiling of copper-stressed Pseudomonas aeruginosa', J. Bacteriol. Vol. 188 , pp. $7242-7256$.

52. Frangipani, E., Slaveykova, V.I., Reimmann, C. and Haas, D. (2008), 'Adaptation of aerobically growing Pseudomonas aeruginosa to copper starvation', J. Bacteriol. Vol. 190, pp. 6706-6717.

53. Chace, K.V., Leahy, D.S., Martin, R., Carubelli, R. et al. (1983), 'Respiratory mucous secretions in patients with cystic fibrosis: Relationship between levels of highly sulfated mucin component and severity of the disease', Clin. Chim. Acta Vol. 132, pp. 143-155.

54. Xia, B., Royall, J.A., Damera, G. et al. (2005), 'Altered O-glycosylation and sulfation of airway mucins associated with cystic fibrosis', Glycobiology Vol. 15, pp. 747-775.

55. Quadroni, M., James, P., Dainese-Hatt, P. and Kertesz, M.A. (1999), 'Proteome mapping, mass spectrometric sequencing and reverse transcription-PCR for characterization of the sulfate starvation-induced response in Pseudomonas aeruginosa PAO1', Eur. J. Biochem. Vol. 266, pp. 986-996.

56. Tralau, T., Vuilleumier, S., Thibault, C., Campbell, B.J. et al. (2007), 'Transcriptomic analysis of the sulfate starvation response of Pseudomonas aeruginosa', J. Bacteriol. Vol. 189, pp. 6743-6750.

57. Aspedon, A., Palmer, K. and Whiteley, M. (2006), 'Microarray analysis of the osmotic stress response in Pseudomonas aeruginosa', J. Bacteriol. Vol. 188 , pp. $2721-2725$.

58. Garay-Arroyo, A., Colmenero-Flores, J.M., Garciarrubio, A. and Covarrubias, A.A. (2000), 'Highly hydrophilic proteins in prokaryotes and eukaryotes are common during conditions of water deficit'. J. Biol. Chem. Vol. 275, pp. 5668-5574.

59. Waters, C.M. and Bassler, B.L. (2005), 'Quorum sensing: Cell-to-cell communication in bacteria', Annu. Rev. Cell Dev. Biol. Vol. 21, pp. 319-346.

60. Juhas, M., Eberl, L. and Tummler, B. (2005), 'Quorum sensing: The power of cooperation in the world of Pseudomonas', Environ. Microbiol. Vol. 7, pp. 459-471.

61. Wagner, V.E., Bushnell, D., Passador, L., Brooks, A.I. et al. (2003), 'Microarray analysis of Pseudomonas aeruginosa quorum-sensing regulons: Effects of growth phase and environment', J. Bacteriol. Vol. 185, pp. 2080-2095.

62. Schuster, M., Lostroh, C.P., Ogi, T. and Greenberg, E.P. (2003), 'Identification, timing, and signal specificity of Pseudomonas aeruginosa quorum-controlled genes: A transcriptome analysis', J. Bacteriol. Vol. 185, pp. 2066-2079.

63. Hentzer, M., Wu, H., Andersen, J.B., Riedel, K. et al. (2003), 'Attenuation of Pseudomonas aeruginosa virulence by quorum sensing inhibitors', EMBO J. Vol. 22, pp. 3803-3815.

64. Schuster, M. and Greenberg, E.P. (2006), 'A network of networks: Quorum-sensing gene regulation in Pseudomonas aeruginosa', Int. J. Med Microbiol. Vol. 296, pp. 73-81.

65. Costerton, J.W., Stewart, P.S. and Greenberg, E.P. (1999), 'Bacterial biofilms: A common cause of persistent infections', Science Vol. 284, pp $1318-1322$

66. Drenkard, E. (2003), 'Antimicrobial resistance of Pseudomonas aeruginose biofilms', Microbes Infect. Vol. 5, pp. 1213-1219.

67. Whiteley, M., Bangera, M.G., Bumgarner, R.E., Parsek, M.R. et al. (2001), 'Gene expression in Pseudomonas aeruginosa biofilms', Nature Vol. 413, pp. 860-864.

68. Bagge, N., Schuster, M., Hentzer, M., Ciofu, O. et al. (2004), 'Pseudomonas aeruginosa biofilms exposed to imipenem exhibit changes in global gene expression and beta-lactamase and alginate production', Antimicrob. Agents Chemother. Vol. 48, pp. 1175-1187.

69. Govan, J.R. and Deretic, V. (1996), 'Microbial pathogenesis in cystic fibrosis: Mucoid Pseudomonas aeruginosa and Burkholderia cepacia', Microbiol. Rev. Vol. 60, pp. 539-574.

70. Firoved, A.M., Boucher, J.C. and Deretic, V. (2002), 'Global genomic analysis of $\mathrm{AlgU}$ (sigma(E))-dependent promoters (sigmulon) in Pseudomonas aeruginosa and implications for inflammatory processes in cystic fibrosis', J. Bacteriol. Vol. 184, pp. 1057-1064.
71. Firoved, A.M. and Deretic, V. (2003), 'Microarray analysis of global gene expression in mucoid Pseudomonas aeruginosa', J. Bacteriol. Vol. 185, pp. 1071-1081.

72. Feldman, M., Bryan, R., Rajan, S., Scheffler, L. et al. (1998), 'Role of flagella in pathogenesis of Pseudomonas aeruginosa pulmonary infection', Infect. Immun. Vol. 66, pp. 43-51.

73. Tart, A.H., Wolfgang, M.C. and Wozniak, D.J. (2005), 'The alternative sigma factor AlgT represses Pseudomonas aeruginosa flagellum biosynthesis by inhibiting expression of fleQ', J. Bacteriol. Vol. 187, pp. 7955-7962.

74. Dasgupta, N., Wolfgang, M.C., Goodman, A.L., Arora, S.K. et al. (2003), 'A four-tiered transcriptional regulatory circuit controls flagellar biogenesis in Pseudomonas aeruginosa', Mol. Microbiol. Vol. 50, pp. 809-824.

75. Kipnis, E., Sawa, T. and Wiener-Kronish, J. (2006), 'Targeting mechanisms of Pseudomonas aeruginosa pathogenesis', Med. Mal. Infect. Vol. 36, pp. 78-91.

76. Knirel, Y.A., Bystrova, O.V., Kocharova, N.A., Zahringer, U. et al. (2006), 'Conserved and variable structural features in the lipopolysaccharide of Pseudomonas aeruginosa', J. Endotoxin Res. Vol. 12, pp. 324-336.

77. Goldberg, J.B., Hatano, K. and Pier, G.B. (1993), 'Synthesis of lipopolysaccharide $\mathrm{O}$ side chains by Pseudomonas aeruginosa $\mathrm{PAO} 1$ requires the enzyme phosphomannomutase', J. Bacteriol. Vol. 175, pp. $1605-1611$

78. Sabra, W.H.L. and Zeng, A.-P. (2003), 'Alterations in the formation of lipopolysaccharide and membrane vesicles on the surface of Pseudomonas aeruginosa PAO1 under oxygen stress conditions', Microbiology Vol. 149, pp. 2789-2795.

79. Worlitzsch, D., Tarran, R., Ulrich, M., Schwab, U. et al. (2003), 'Effects of reduced mucus oxygen concentration in airway Pseudomonas infections of cystic fibrosis patients', J. Clin. Invest. Vol. 109, pp. 317-325.

80. Hassett, D.J. (1996), 'Anaerobic production of alginate by Pseudomonas aeruginosa: Alginate restricts diffusion of oxygen', J. Bacteriol. Vol. 178, pp. $7322-7325$.

81. Firoved, A.M., Ornatowski, W. and Deretic, V. (2004), 'Microarray analysis reveals induction of lipoprotein genes in mucoid Pseudomonas aeruginosa: Implications for inflammation in cystic fibrosis', Infect. Immun. Vol. 72, pp. 5012-5018.

82. Burrowes, E., Baysse, C., Adams, C. and O'Gara, F. (2006), 'Influence of the regulatory protein RsmA on cellular functions in Pseudomonas aeruginosa PAO1, as revealed by transcriptome analysis', Microbiology Vol. 152, pp. 405-418.

83. Burrowes, E., Abbas, A., O’Neill, A., Adams, C. et al. (2005), 'Characterisation of the regulatory RNA RsmB from Pseudomonas aeruginosa PAO1', Res. Microbiol. Vol. 156, pp. 7-16.

84. Pessi, G., Williams, F., Hindle, Z., Heurlier, K. et al. (2001), 'The global posttranscriptional regulator RsmA modulates production of virulence determinants and $\mathrm{N}$-acylhomoserine lactones in Pseudomonas aeruginosa', J. Bacteriol. Vol. 183, pp. 6676-6683.

85. Ichikawa, J.K., Norris, A., Bangera, M.G., Geiss, G.K. et al. (2000), 'Interaction of Pseudomonas aeruginosa with epithelial cells: Identification of differentially regulated genes by expression microarray analysis of human cDNAs', Proc. Natl. Acad. Sci. USA Vol. 97, pp. 9659-9664.

86. Frisk, A., Schurr, J.R., Wang, G., Bertucci, D.C. et al. (2004), 'Transcriptome analysis of Pseudomonas aeruginosa after interaction with human airway epithelial cells', Infect. Immun. Vol. 72, pp. 5433-5438.

87. Chugani, S. and Greenberg, E.P. (2007), 'The influence of human respiratory epithelia on Pseudomonas aeruginosa gene expression', Microb. Pathog. Vol. 42, pp. 29-35.

88. Jander, G., Rahme, L.G. and Ausubel, FM. (2000), 'Positive correlation between virulence of Pseudomonas aeruginosa mutants in mice and insects', J. Bacteriol. Vol. 182, pp. 3843-3845.

89. Mahajan-Miklos, S., Tan, M.W., Rahme, L.G. and Ausubel, F.M. (1999), 'Molecular mechanisms of bacterial virulence elucidated using a Pseudomonas aeruginosa-Caenorhabditis elegans pathogenesis model', Cell Vol. 96, pp. 47-56.

90. Rahme, L.G., Tan, M.W., Le, L., Wong, S.M. et al. (1997), 'Use of model plant hosts to identify Pseudomonas aeruginosa virulence factors', Proc. Natl. Acad. Sci. USA Vol. 94, pp. 13245-13250. 